\title{
Krebs-Screening-Programme: Methodische Grundlagen und erste Erfahrungen mit dem Hautkrebs-Screening
}

\author{
Andreas Stang $^{\mathrm{a}^{*}} \quad$ Matthias Augustin $^{\mathrm{b}^{*}}$ \\ a Institut für Klinische Epidemiologie (IKE), Medizinische Fakultät der Martin-Luther Universität Halle-Wittenberg, \\ ${ }^{\mathrm{b}}$ Institut für Versorgungsforschung in der Dermatologie und bei Pflegeberufen, Universitätsklinikum Hamburg-Eppendorf, Deutschland
}

\section{Krebs-Früherkennung}

Die Einführung von Krebs-Screening-Programmen beruht auf der Annahme, dass durch eine frühe Diagnose und erfolgreiche Therapie von Krebsvorstufen oder frühen Tumorstadien effektivere und weniger belastende Therapien zur Verfügung stehen, die in der Lage sind, die tumorspezifische Mortalität zu senken. Als wesentliche Ziele der Früherkennung chronischer Erkrankungen nannte der berühmte Alan S. Morrison 1992 die Senkung der Inzidenz, der krankheitsspezifischen Mortalität und Gesamtmortalität sowie die Verbesserung der Lebensqualität [1].

Auch bei Erfüllung der für eine hohe Akzeptanz bekannten Voraussetzungen (Tab. 1) kann durch Früherkennungsmaßnahmen die Inzidenz invasiver Tumoren allerdings nur dann reduziert werden, wenn wie beim kolorektalen Karzinom, Mammakarzinom oder Melanom die Karzinogenese über mehrere Stufen verläuft und mögliche Krebs-Vorstufen oder frühe Tumorstadien erfolgreich behandelt werden können.

Die beobachtbare Senkung der krankheitsspezifischen Mortalität in einer gescreenten Population hängt vom Anteil an der Gesamtbevölkerung, von der Effektstärke der Mortalitätsreduktion sowie von der krankheitsspezifischen Mortalität vor Beginn des Screenings ab. Wenn beispielsweise eine Krebserkrankung eine sehr niedrige krankheitsspezifische Mortalität in der Bevölkerung aufweist, lässt sich eine weitere Senkung dieser Mortalität statistisch nur in sehr großen Bevölkerungen zeigen, da die statistische Macht (Power) ansonsten nicht ausreicht, um einen Effekt zu zeigen.

Eine Senkung der Gesamtmortalität durch aktives KrebsScreening großer Populationen kann wahrscheinlich nicht einmal bei besonders häufigen Tumoren erwartet werden, da die durch eine erfolgreiche Krebs-Früherkennung und früh-

*A. Stang: «Krebs-Früherkennung»; M. Augustin: «Erste Erfahrungen mit dem deutschen Hautkrebs-Screening» zeitige Therapie zu verhindernden Krebs-Todesfälle nur einen kleinen Teil der Gesamt-Todesfälle ausmachen.

\section{Nutzenbewertung}

Vor dem Hintergrund begrenzter finanzieller Ressourcen sollte aus gesundheitspolitischen Gründen der Nutzen eines Screening-Programms in regelmäßigen Abständen bewertet werden; dabei sollte auch der Einfluss von Faktoren berücksichtigt werden, die möglicherweise zu einer Fehlinterpretation der Daten führen können. Intuitiv überschätzt wird die Wirksamkeit des Krebs-Screenings vor allem durch den Lead Time Bias. Dies bedeutet, dass die infolge des früheren Diagnosezeitpunkts verlängerte Überlebenszeit als Vorteil bewertet wird, obwohl - bei Fehlen einer wirksamen Frühtherapie - die Mortalitätsrate im Vergleich zur Situation ohne Früherkennung unverändert ist. Valide Aussagen zur Effektivität eines Screenings sind daher nur über einen Vergleich der Mortalitätsraten vor und nach Einführung der Früherkennung bzw. zwischen vergleichbaren Populationen mit und ohne Früherkennung möglich (Abb. 1).

Der Nutzen einer Früherkennungsmaßnahme kann durch den Length Time Bias überschätzt werden; damit wird die Beobachtung beschrieben, dass bei regelmäßigen Untersuchungen durch das Screening im Durchschnitt eher langsam wachsende Tumoren aufgedeckt werden. Diese sind aber meist weniger aggressiv und haben eine günstigere Prognose als schnell progrediente Tumoren, die bereits innerhalb der üblichen Screening-Intervalle symptomatisch werden [2].

Tab. 1. Voraussetzungen für die Akzeptanz von Früherkennungsuntersuchungen in der Bevölkerung

- Untersuchung risikoarm, wenig invasiv, schmerzarm

- Untersuchung schnell durchführbar und ubiquitär im Angebot

- Teilnahme am Screening kostenfrei

- Hohe Qualität des Screenings

- Therapeutische Konsequenzen positiver Screening-Ergebnisse

\begin{tabular}{ll}
\hline KARGER & @ 2011 S. Karger GmbH, Freiburg \\
0378-584X/11/3414-0002\$38.00/0 \\
Fax+497614520714 & Accessible online at: \\
Information@Karger.de & www.karger.com/onk \\
www.karger.com &
\end{tabular}




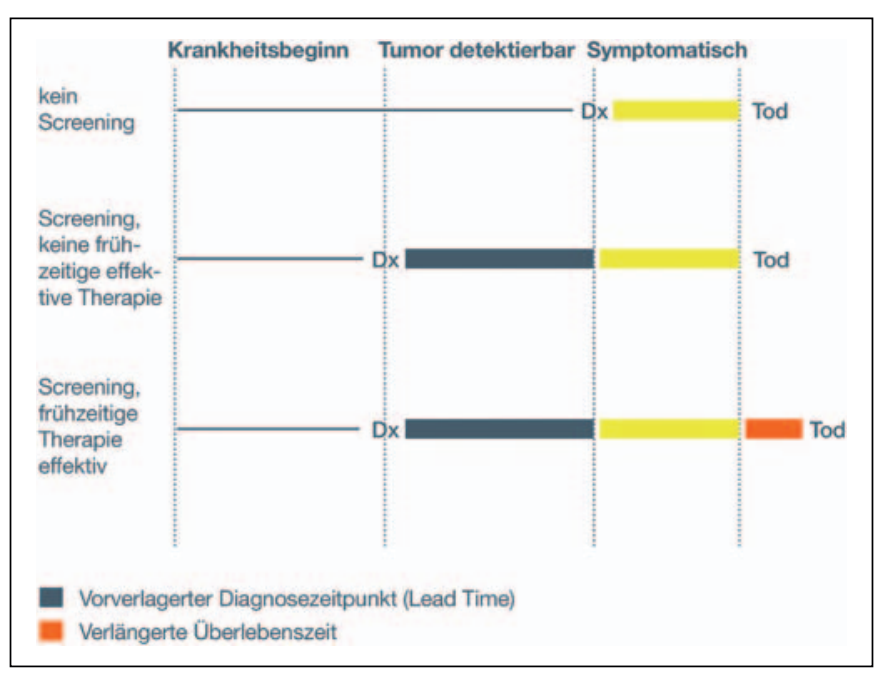

Abb. 1. Illustration des Lead Time Bias (Dx: Diagnosezeitpunkt).

Als Sonderform des Length Time Bias wird die Overdiagnosis angesehen. Dabei handelt es sich um Läsionen mit allen histopathologischen Zeichen eines bösartigen Tumors, der allerdings zu Lebzeiten des Patienten keine Symptome hervorruft und damit klinisch ohne Bedeutung ist. Nach Daten aus Autopsie-Studien, Studien mit Wait-and-see-Strategie sowie Modellrechnungen aus Beobachtungsdaten ist mit einer Overdiagnosis vor allem beim Prostatakarzinom zu rechnen, wird aber auch beim Mammakarzinom und anderen soliden Tumoren berichtet. Ein Sonderfall der Overdiagnosis sind regressionsfähige Malignome. Hierbei handelt es sich um Malignome, die sich ohne Therapie komplett selbst zurückbilden.

Der Healthy Volunteer Bias beschreibt die Beobachtung, dass Teilnehmer an Screening-Programmen möglicherweise gesundheitsbewusster leben und aufgrund weniger Risikofaktoren bei einer Tumorerkrankung möglicherweise generell eine bessere Prognose haben.

Veränderungen im Gesundheitsbewusstsein und Fortschritte in der Therapie können die Nutzenbewertung einer Krebs-Früherkennung beeinflussen, wie eine kürzlich publizierte Diskussion am Beispiel des Mammakarzinoms zeigte [3]. Auch ohne Screening ist beim Mammakarzinom die krankheitsspezifische Mortalitätsrate zwischen 1980-1986 und 20002006 deutlich gesunken. Dafür gibt es mindestens 2 Erklärungen: Zum einen hat es in den letzten 20 Jahren erhebliche therapeutische Fortschritte in der Behandlung des invasiven Mammakarzinoms gegeben. Weiterhin wird angenommen, dass sich aufgrund der zunehmenden Awareness in der Bevölkerung und bei den Ärzten Patientinnen mit Mammakarzinom heute in früheren Stadien beim Arzt vorstellen. Diese Faktoren erschweren die Nutzenbewertung des MammographieScreenings, da die meisten randomisierten Studien zum Mammographie-Screening in den 1980er-Jahren durchgeführt wurden; zu der Zeit war die Effektivität der Behandlung von Mammakarzinomen deutlich schlechter als heutzutage. Eine

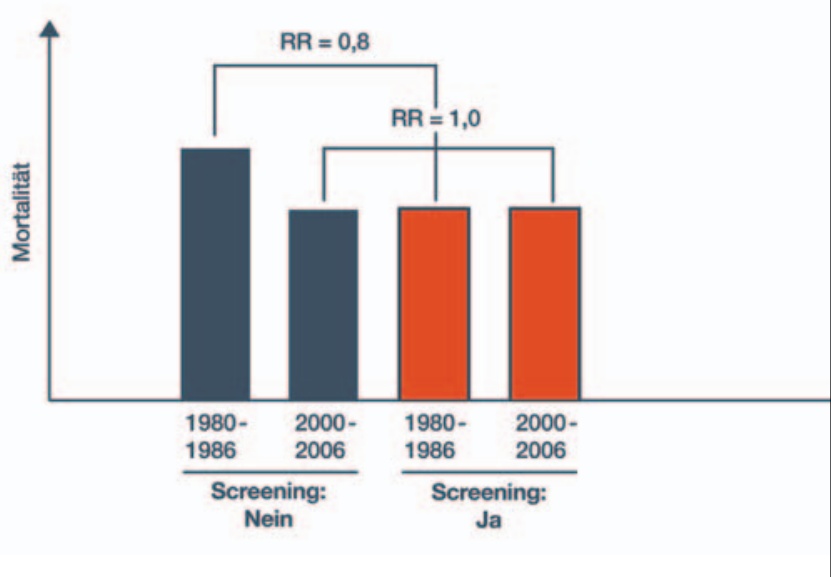

Abb. 2. Vergleich der krankheitsspezifischen Mortalität in einer NichtScreening- und einer Screening-Gruppe in der alten Ära der Brustkrebsbehandlung (hohe Mortalität ohne Screening) und der neuen Ära der Brustkrebsbehandlung (niedrigere Mortalität ohne Screening) (RR: relatives Risiko, am Mammakarzinom zu versterben).

Reduktion der krankheitsspezifischen Mortalität durch Mammographie-Screening konnte damit einfacher gezeigt werden als heutzutage, wo auch ohne Screening die Behandlung des Mammakarzinoms im Durchschnitt sehr wirksam ist (Abb. 2).

Die Prävalenz von nicht diagnostizierten, durch Screening detektierbaren Mammakarzinomen hängt auch vom Ausmaß der vorangegangenen Screening-Aktivitäten ab. Die Daten des Saarländischen Krebsregisters aus dem Jahr 1997 (also lange vor Einführung des gesetzlichen Mammographie-Screenings) zeigten eine ähnliche stadienspezifische Verteilung von Mammakarzinomen wie in den Niederlanden etwa 10 Jahre nach Einführung des Mammographie-Screenings [4]. Dieser Befund ist im Wesentlichen auf das «graue» (opportunistische) Mammographie-Screening zurückzuführen.

Auch die vorgesehene Nutzenbewertung des gesetzlichen Screenings auf Hautkrebs dürfte dadurch erschwert werden, dass in vielen Hautarzt-Praxen bereits vor Einführung des Programms entsprechende Untersuchungen als «graues Screening» durchgeführt wurden.

\section{Hautkrebs-Screening: Erste Erfahrungen aus der täglichen Praxis}

Zum 1. Juli 2008 wurde in Deutschland das gesetzliche Hautkrebs-Screening (gHKS) als Regelleistung der Gesetzlichen Krankenversicherungen (GKV) eingeführt [5]. Versicherte ab 35 Jahren können seitdem alle 2 Jahre eine Früherkennungsuntersuchung auf Hautkrebs in Anspruch nehmen, wobei vom Gemeinsamen Bundesausschuss (GBA) aufgrund der Schwere bzw. hohen Inzidenz das maligne Melanom, Spinaliom und Basaliom als Zieldiagnosen ausgewählt wurden [6]. 
Zum Hautkrebs-Screening leistungsberechtigt sind Dermatologen sowie qualifiziert fortgebildete Allgemeinmediziner, praktische Ärzte und gebietsfreie Ärzte. Damit erhalten die deutschen Dermatologen sowie die weiteren zum Screening berechtigten Kassenärzte in Deutschland einen umfassenden Versorgungsauftrag.

Da die wissenschaftliche Evidenz zum Zusammenhang von Hautkrebs-Screening und Mortalitäts- und/oder Morbiditätssenkung derzeit unzureichend ist, soll der Erfolg der Früherkennung spätestens 5 Jahre nach Inkrafttreten der entsprechenden Richtlinienänderung geprüft werden. Zur Evaluation der Wirksamkeit des gHKS wurde allerdings keine prospektive, kontrollierte Studie vorgesehen. Mit erheblicher Verzögerung zum Start des gHKS wurde erst im November 2010 die Evaluation des gesetzlichen Screenings ausgeschrieben. Diese wird allerdings lediglich auf einen schmalen Sekundärdatensatz zurückgreifen können, der infolge von Dokumentationsproblemen und unzureichender Konzeption nur bedingt verwertbar sein wird. Die Deutsche Dermatologische Gesellschaft und der Berufsverband der Deutschen Dermatologen haben auf diese absehbare Lücke bereits im Jahr 2008 reagiert und ein umfangreiches Begleitforschungsprogramm gestartet.

\section{Auswirkungen des gHKS auf Hautarztpraxen}

Seit 2009 werden die Auswirkungen des gHKS auf die hautärztliche Versorgung untersucht. Dazu werden jeweils im Frühjahr standardisierte Fragebögen an bundesweit 2000 Hautarztpraxen versendet. Die Daten werden deskriptiv sowie anhand multipler Regressionen ausgewertet. Bei einer Rücklaufquote von 34,7\% (2009) bzw. 32,8\% (2010) konnten bis Juni 2010 die Angaben aus 693 (2009) bzw. 656 (2010) Evaluationsbögen analysiert werden [7].

Pro Hautarztpraxis wurden pro Quartal durchschnittlich 354 bzw. 341 Hautkrebs-Screenings durchgeführt, wobei die mittlere Vergütung 21,50 € (2009) bzw. 22,10 € (2010) betrug. 78\% bzw. 71\% der Praxen sahen seit Einführung des gHKS eine Zunahme dieser Untersuchung um durchschnittlich 36,7\% (2009) bzw. 47\% (2010). Sowohl 2009 als auch 2010 führten 54\% der Praxen das HKS als Kassenleistung in Verbindung mit individuellen Gesundheitsleistungen (IGeL) durch, 38\% bzw. 30\% als alleinige Kassenleistung und $8 \%$ bzw. $6 \%$ als reine IGeL.
Seit Einführung des gHKS stieg in $85 \%$ (2009) bzw. $82 \%$ (2010) der Praxen die Anzahl operativer Eingriffe um jeweils durchschnittlich 23\%, das Aufkommen an Arzneimittelverordnungen nahm in $40 \%$ bzw. $39 \%$ der Praxen um durchschnittlich 7\% bzw. 6\% zu. Der Anteil der mit der Einführung des Hautkrebs-Screenings als GKV-Regelleistung zufriedenen Praxen stieg von 32\% (2009) auf 47\% (2010), während der Anteil der mit dem gHKS unzufriedenen Praxen von $40 \%$ auf $23 \%$ abnahm. Bei den Präferenzen nach der Abrechnungsmodalität befragt, würden $29 \%$ bzw. $25 \%$ das HKS als reine GKV-Leistung, 29\% bzw. 21\% als reine IGeL und 42\% bzw. 54\% als kombinierte GKV-Leistung plus IGeL bevorzugen. 2009 gaben $70 \%$ der befragten Hautarztpraxen an, dass sich in Deutschland mit Einführung des gHKS die Versorgung von Patienten mit Hauttumoren verbessert habe, 2010 beurteilten $80 \%$ die Versorgung als verbessert.

\section{Fazit und Ausblick}

Die Dermatologen haben mit dem gHKS einen wichtigen gesetzlichen Versorgungsauftrag angenommen, dessen Umsetzung jedoch noch optimierter Rahmenbedingungen bedarf. Die in Hautarztpraxen mittels standardisierter Fragebögen erhobenen Daten zeigen für den Vergleich der Jahre 2009 und 2010 weitgehend stabile Ergebnisse bei einer Zunahme der Akzeptanz des gHKS. Die Ergebnisse der weiteren Versorgungsstudien werden erst 2011 erwartet.

Zur Bewertung des Nutzens, der Qualität und Effizienz des gHKS sind mehrere, unterschiedlich konzipierte Studien erforderlich, die auf Primärdaten- und Sekundärdatenauswertungen beruhen und in verschiedenen Bereichen der Versorgung erhoben werden sollten. In Verbindung mit den - bislang noch nicht publizierten - Daten des Modellvorhabens in Schleswig-Holstein könnte das gesamte Forschungsaufkommen eine valide Datenbasis für eine umfassende Bewertung des gHKS liefern.

\section{Disclosure Statement}

Die Autoren waren Referenten beim «1. Interdisziplinäres Expertenforum Hautkrebs», das von Bristol-Myers Squibb unterstützt wurde.

\section{Literatur}

1 Morrison AS: Screening in Chronic Disease, ed 2. New York, Oxford University Press, 1992.

2 Kramer BS, Croswell JM: Cancer screening: the clash of science and intuition. Annu Rev Med 2009; 60:125-137.

3 Welch HG: Screening mammography - a long run for a short slide? New Engl J Med 2010;363:12761278 .

4 Stegmaier C, Sonderauswertung, persönliche Kommunikation.

\footnotetext{
5 Bundesministerium für Gesundheit: Bekanntmachung [1430 A] eines Beschlusses des Gemeinsamen Bundesausschusses über eine Änderung der Krebsfrüherkennungs-Richtlinien: HautkrebsScreening vom 15. November 2007. BAnz Nr. 37 (S. 871) vom 6.3.2008.

6 Gemeinsamer Bundesausschuss: Tragende Gründe zum Beschluss des Gemeinsamen Bundesausschusses über eine Änderung der KrebsfrüherkennungsRichtlinien: Hautkrebs-Screening vom 15. November 2007. Siegburg, 2007. www.g-ba.de/downloads/ 40-268-482/2007-11-15-KFU-Hautkrebsscreening_ TrG.pdf.
}

Augustin M, Blome C, Rustenbach SJ, et al.: Einführung des Hautkrebs-Screening als Regelleistung der gesetzlichen Krankenkassen: Erste Daten zu den Auswirkungen auf die dermatologische Versorgung. J Dtsch Dermatol Ges 2010;8:674-680. 